\title{
Dois Critérios de Seleção na Pré-Desmama em Bovinos da Raça Gir. 1. Estimativas de Parâmetros Genéticos ${ }^{1}$
}

\author{
Carolina Amália de Souza Dantas Muniz², Roberto Carvalheiro ${ }^{3}$, Luiz Alberto Fries ${ }^{3,4}$, \\ Sandra Aidar de Queiroz ${ }^{5}$
}

\begin{abstract}
RESUMO - Dados de 16.592 animais da raça Gir, provenientes do controle de desenvolvimento ponderal da Associação Brasileira dos Criadores de Zebu, nascidos no período de 1978 a 1994, criados em diversas condições de ambiente no Brasil, foram usados para estimar parâmetros genéticos e ambientais das características ganho médio diário do nascimento à desmama (GMD) e dias para atingir $160 \mathrm{~kg}$ do nascimento à desmama (D160). As estimativas dos componentes de variância e herdabilidade foram obtidas pelo método da máxima verossimilhança restrita por modelos animais uni-característica. Foram utilizados dois modelos - o modelo 1, com grupo de contemporâneos (GC) como efeito fixo e com efeitos genético aditivo de animal e materno, efeito de ambiente permanente materno e erro como aleatórios; e modelo 2, com os mesmos efeitos do modelo 1, mas com covariância genética entre os efeitos direto e materno igual a zero $\left(\sigma_{\mathrm{am}}=0\right)$. As estimativas de herdabilidade, utilizando-se o Modelo 1, foram iguais a 0,11; 0,04; 0,11 e 0,07 para GMD (efeito direto), GMD (efeito materno), D160 (efeito direto) e D160 (efeito materno), respectivamente. Para o Modelo 2, as estimativas de herdabilidade foram: 0,12; 0,05; 0,10 e 0,05 para GMD (efeito direto), GMD (efeito materno), D160 (efeito direto) e D160 (efeito materno). A diferença entre os modelos pela não inclusão da estrutura de covariância entre os efeitos direto e materno $\left(\sigma_{\mathrm{am}}=0\right)$, neste trabalho, não foi significativa. A contribuição da variância residual (como porcentagem da variação fenotípica total) foi de $76 \%$ para GMD e de $80 \%$ para D160, para os dois modelos, indicando a necessidade da melhoria do ambiente de criação, assim como a padronização e formação de lotes de manejo, o que reflete na possibilidade de melhor definição de grupo de contemporâneos.
\end{abstract}

Palavras-chave: correlação genética entre efeitos direto e materno, dias para atingir 160 do nascimento à desmama, ganho médio do nascimento à desmama, herdabilidade do efeito direto, herdabilidade do efeito materno

\section{Two Pre-Weaning Selection Criteria in Gyr Cattle. 1. Genetic Parameter Estimates}

\begin{abstract}
A data set with 16,592 records from the Brazilian Zebu Breeders Association obtained from 1978 to 1994 , was used to estimate genetic parameters for preweaning average daily gain (ADG) and number of days to gain $160 \mathrm{~kg}$ (D160) in Gyr cattle. The (co)variance components and heritabilities were estimated by the restricted maximum likelihood derivative free method. Two models were used: the first one considered contemporary group (CG) as fixed effect and additive direct and maternal genetic effects, maternal permanent environment and error as random effect. Model 2 considered the same effects of 1 but the covariance between the genetic direct and maternal effects was assumed to be zero. Estimated heritability values using model 1 were $0.11 ; 0.04 ; 0.11$ and 0.07 for ADG (direct effect), ADG (maternal effect), D160 (direct effect), and D160 (maternal effect), respectivelly, and 0.12, 0.05, 0.10 and 0.05 considering Model 2 for ADG (direct effect), ADG (maternal effect), D160 (direct effect), and D160 (maternal effect), respectivelly. The likelihood ratio test was used to compare these models and were not significant. The proportional residual variances were 76 and $80 \%$ for ADG and D160, respectively for both models. The hight values determined for the residual variance indicate the need to improve the breeding environment, such as standartization of manegement groups in order to get definition of contemporary group.
\end{abstract}

Key Words: average daily gain from birth to weaning, days to gain $160 \mathrm{~kg}$ from birth to weaning, direct heritability, directmaternal genetic correlation, maternal heritability

\section{Introdução}

A bovinocultura de corte tropical e subtropical caracteriza-se, basicamente, por sistemas de produção em pastejo, que se constituem, em sua maioria, das fases de cria, recria e terminação. A fase de cria compreende todos os processos de produção envolvi- dos desde a escolha e cuidado dos progenitores até o desmame do produto. Segundo Fries (1996), cerca de 2/3 dos custos de produção de carne bovina estão associados à fase de cria, compreendendo a recria de fêmeas de reposição até os processos de gestação, lactação e desmame. Portanto, as características da pré-desmama são de grande importância econômica,

\footnotetext{
${ }^{1}$ Parte da tese de doutorado em Zootecnia da primeira autora/Produção Animal na FCAVJ - Unesp - Jaboticabal - SP. Projeto financiado pelo CNPq.

${ }^{2}$ Centro Universitário das Faculdades Metropolitanas Unidas - UniFMU - São Paulo - SP (muniz_ca@ig.com.br).

${ }^{3}$ GenSys Consultores Associados S/C Ltda - Porto Alegre - RS.

${ }^{4}$ Lagoa da Serra Ltda - Sertãozinho - SP - Brasil.

${ }^{5}$ Departamento de Zootecnia - FCAV - UNESP. Via de acesso Prof. Paulo Donato Castelane, CEP: 14884-900, Jaboticabal - SP. Bolsista do CNPq.
} 
uma vez que são de fácil mensuração e podem ser utilizadas para avaliar tanto o potencial genético de crescimento do bezerro como a habilidade materna da vaca.

O desenvolvimento do bezerro até a desmama é freqüentemente medido pelo ganho médio diário do nascimento à desmama (GMD) e pelo peso à desmama ajustado para a idade do animal. O cálculo do GMD considera o ganho de peso no período avaliado no numerador e a diferença em dias entre as pesagens consecutivas no denominador, gerando a taxa ou velocidade de crescimento, expressa como ganho médio diário. Segundo Giannoni \& Giannoni (1989), as principais maneiras de se avaliar a velocidade de crescimento, desde que as condições ambientais sejam corrigidas para todos os indivíduos em controle, são: a) a contagem do número de dias necessários para o animal atingir determinado peso e b) a verificação do ganho em peso em um período de tempo fixo. Os critérios de seleção para crescimento são, principalmente, os pesos registrados em determinadas idades.

Fries et al. (1996) propuseram a característica número de dias para o animal ganhar determinado peso, no caso do período pré-desmama, o número de dias para o animal ganhar $160 \mathrm{~kg}$ entre o nascimento e a desmama (D160). Um dos objetivos deste critério (D160) seria fornecer aos criadores menor espaço de tempo para produzir determinadas unidades de mercado, em vez de buscar animais cada vez mais pesados, indefinidamente.

O desempenho do bezerro até a desmama é conseqüência de seu potencial para crescimento e da habilidade materna de sua mãe. A habilidade materna compreende uma série de fatores - desenvolvimento intra-uterino, nascimento, cuidados da mãe com a cria e amamentação. Fries \& Albuquerque (1998) destacaram a produção de leite como o principal fator a afetar o desenvolvimento do bezerro, com correlação de $60 \%$, em média, entre produção de leite da vaca e peso do bezerro à desmama.

A relação entre fenótipo da vaca e fenótipo do bezerro tem sido documentada em pesquisas com gado de corte, gerando dúvidas a respeito dos valores obtidos para as correlações entre efeitos genéticos direto e materno, quando se estimam componentes de variância. Diversos trabalhos envolvendo bovinos de corte estimaram correlações genéticas negativas e altas entre efeitos genéticos materno e direto para características pré-desmama (Cardoso et al., 2001; Ribeiro et al., 2001; Pimenta Filho et al., 2001).
Entretanto, Robinson (1996) encontrou evidências de que as estimativas de $r_{a m}$ negativas foram mais consequiência da variação adicional entre touros ou da interação touro $x$ ano do que uma real relação genética negativa.

Schaeffer (1993), utilizando um exemplo numérico, demonstrou que a correlação genética negativa entre efeitos genéticos direto e materno pode ser resultado da estimativa do efeito materno, como função linear do efeito direto. Como exemplo, esse autor citou que o efeito materno foi estimado para touros, mesmo que não tivessem qualquer filha desmamando um bezerro. Esses touros sem filhas apresentam correlação entre os efeitos direto e materno iguais a menos um $(-1,0)$, porque não existe observação para a estimativa do efeito materno, sendo função apenas do efeito direto.

Em revisão sobre o assunto, Fries \& Albuquerque (1998) sugeriram que ideal seria usar covariância igual a zero para efeitos direto e materno, pelo menos até que se obtenham informações suficientes e que se conheça melhor a relação entre esses efeitos.

Neste estudo, foram estimados parâmetros genéticos para as características ganho médio diário do nascimento à desmama (GMD) e dias para ganhar 160 $\mathrm{kg}$ do nascimento à desmama (D160), em bovinos da raça Gir, utilizando-se dois modelos, um que considera a estrutura de covariância entre os efeitos genéticos direto e materno e outro modelo em que a covariância entre os efeitos genéticos direto e materno foi considerada igual a zero.

\section{Material e Métodos}

Os dados utilizados neste estudo foram provenientes do controle de desenvolvimento ponderal da raça Gir, da Associação Brasileira de Criadores de Zebu (ABCZ), de animais nascidos no período de 1978 a 1994, criados em diversas condições no Brasil, em um total de 16.592 observações de ganho médio diário do nascimento à desmama (GMD) e de dias para ganhar $160 \mathrm{~kg}$ do nascimento à desmama (D160). O GMD foi pré ajustado para a idade e data juliana de nascimento do bezerro e para a idade da vaca conforme sexo do bezerro, utilizando-se fatores de correção determinados especificamente para este conjunto de dados (Pelicioni et al., 2002). As observações de D160 foram obtidas pela equação: D160 = 160/GMD pré-ajustado. O grupo de contemporâneos (GC) foi formado com as seguintes variáveis: ano de 
nascimento, estação de nascimento, criador e fazenda (rebanho), sexo, raça (Gir mocho e Gir padrão), categoria (LA - livro aberto e LF - livro fechado) e grupo de regime alimentar. Grupos de contemporâneos com menos de cinco animais foram eliminados.

As estações de nascimento consideradas foram primavera (22 set - $21 \mathrm{dez})$, verão ( $22 \mathrm{dez}-21$ - mar), outono (22 mar - 21 jun) e inverno (22 jun -21 set). Os regimes alimentares considerados foram (1) pastagem; (2) animal semi-estabulado; e (3) animal estabulado. Foi verificado, por meio do programa MILC (Matriz de Incidência, Laços genéticos e Conectabilidade), desenvolvido por Fries \& Roso (1997), o grau de conectabilidade dos dados, entre diferentes grupos de contemporâneos. O programa apresentado por Fries (1998), faz a contagem dos laços genéticos diretos existentes entre grupos de contemporâneos, por meio de touros e vacas com progênie nesses grupos. Assim, existe um laço genético, que é uma medida de conectabilidade, direto entre um grupo de contemporâneos e outro quando houver um touro (ou vaca) estimável (com vários filhos em diferentes grupos de contemporâneos) com pelo menos uma progênie controlada em cada um desses grupos. Os registros que foram considerados desconectados pelo programa foram excluídos das análises.

As estimativas dos componentes de variância foram obtidas pelo método da Máxima Verossimilhança Restrita (REML), por meio de modelos animais (MA) uni-característica, utilizando-se o programa computacional multiple-trait derivative-free restricted maximum likelihood (MTDFREML), segundo Boldman et al. (1993), com equações de modelos mistos (EMM).

As estimativas dos componentes de variância foram obtidas utilizando-se dois modelos:

Modelo 1: $\mathrm{y}=\mathrm{Xb}+\mathrm{Za}+\mathrm{Mm}+\mathrm{Wp}+\mathrm{e}, \operatorname{com}\left(\sigma_{\mathrm{am}}\right) \neq 0$

Modelo 2: $\mathrm{y}=\mathrm{Xb}+\mathrm{Za}+\mathrm{Mm}+\mathrm{Wp}+\mathrm{e}, \operatorname{com}\left(\sigma_{\mathrm{am}}\right)=0$

em que: $\mathbf{y}=$ etor das observações pré-ajustadas (GMD ou D160); $\mathbf{X}=$ matriz de incidência associada aos efeitos fixos; $\mathbf{b}=$ =vetor dos efeitos fixos (grupo de contemporâneos); $\mathbf{Z}, \mathbf{M}, \mathbf{W}=$ matrizes de incidência associadas aos efeitos aleatórios; $\mathbf{a}=$ vetor do efeito genético aditivo direto (AD) do animal (aleatório); $\mathbf{m}=$ vetor do efeito genético aditivo materno (AM), (aleatório); $\mathbf{p}=$ vetor do efeito de ambiente permanente (AP), (aleatório); $\mathbf{e}=$ vetor dos resíduos aleatórios, não observáveis; $\left(\sigma_{\mathrm{am}}\right)=$ é a covariância entre efeitos genéticos diretos e maternos.

Nos modelos considerados para as análises genéticas, têm-se as seguintes pressuposições:

$$
\mathrm{E}=\left[\begin{array}{c}
\mathrm{y} \\
\mathrm{a} \\
\mathrm{m} \\
\mathrm{p} \\
\mathrm{e}
\end{array}\right]=\left[\begin{array}{c}
\mathrm{Xb} \\
0 \\
0 \\
0 \\
0
\end{array}\right]
$$

$$
\mathrm{VAR}=\left[\begin{array}{c}
\mathrm{a} \\
\mathrm{m} \\
\mathrm{p} \\
\mathrm{e}
\end{array}\right]=\left[\begin{array}{cccc}
\sigma_{\mathrm{a}}^{2} \mathrm{~A} & \sigma_{\mathrm{am}} \mathrm{A} & 0 & 0 \\
\sigma_{\mathrm{am}} \mathrm{A} & \sigma_{\mathrm{m}}^{2} \mathrm{~A} & 0 & 0 \\
0 & 0 & \sigma_{\mathrm{P}}^{2} \mathrm{I}_{\mathrm{C}} & 0 \\
0 & 0 & 0 & \sigma_{\mathrm{e}}^{2} \mathrm{I}_{\mathrm{N}}
\end{array}\right]
$$

Para o Modelo 1, e para o Modelo $2\left(\sigma_{\text {am }}\right)=0$ em que: $\mathrm{A}=$ numerador da matriz de parentesco; $\mathrm{N}=$ número de registros; $\mathrm{I}=$ matriz identidade; $\mathrm{C}=$ número de vacas; $\sigma_{\mathrm{a}}^{2}=$ variância genética aditiva direta; $\sigma_{\mathrm{m}}^{2}=$ variância genética aditiva materna; $\sigma_{\mathrm{am}}=$ covariância entre efeitos genéticos diretos e maternos; $\sigma_{\mathrm{p}}^{2}=$ variância de ambiente permanente; $\sigma_{\mathrm{e}}^{2}=$ variância residual.

As estimativas dos coeficientes de herdabilidade dos efeitos genéticos aditivo direto $\left(\mathbf{h}_{\mathbf{D}}^{2}\right)$ e materno $\left(\mathbf{h}_{\mathbf{M}}^{2}\right)$ e efeito genético total $\left(\mathbf{h}_{\mathbf{T}}^{2}\right)$ foram obtidas de acordo com: as fórmulas:

$\mathbf{h}_{\mathbf{D}}^{2}=\sigma_{\mathrm{a}}^{2} / \sigma_{\mathrm{F}}^{2}, \mathbf{h}_{\mathrm{M}}^{2}=\sigma_{\mathrm{m}}^{2} / \sigma_{\mathrm{F}}^{2}, \mathbf{h}_{\mathrm{T}}^{2}=\frac{\sigma_{\mathrm{a}}^{2}+0,5 \sigma_{\mathrm{m}}^{2}+1,5 \sigma_{\mathrm{am}}}{\sigma_{\mathrm{F}}^{2}}$ respectivamente, em que: $\sigma_{\mathrm{F}}^{2}=\sigma_{\mathrm{a}}^{2}+\sigma_{\mathrm{m}}^{2}+\sigma_{\mathrm{p}}^{2}+\sigma_{\mathrm{e}}^{2}$, em que $\sigma_{\mathrm{F}}^{2}$ é a variância fenotípica total e $\sigma_{\mathrm{am}}$, a covariância entre os efeitos genéticos aditivos direto e materno.

A estimativa da herdabilidade do efeito genético total $\left(\mathrm{h}_{\mathrm{t}}^{2}\right)$, foi obtida de acordo com Willham (1972). As covariâncias entre os efeitos genéticos e ambientais (permanentes e temporários), bem como entre os efeitos ambientais de diferentes animais, foram assumidas como zero.

A diferença na qualidade do ajuste entre os mode$\operatorname{los} 1\left(\sigma_{\mathrm{am}} \neq 0\right)$ e $2\left(\sigma_{\mathrm{am}}=0\right)$ foi verificada pelo teste da Razão de Verossimilhança (Likelihood Ratio Test) (Hogg \& Craig, 1995), comparando-se o aumento causado no valor de -2log L do modelo 2 pela retirada de um parâmetro, em relação ao valor de $-2 \log \mathrm{L}$ do modelo 1, a uma distribuição qui-quadrado com grau de liberdade e probabilidade de erro de $5 \%$. 
Na Tabela 1, pode ser observada a estrutura do conjunto de dados para GMD e D160.

Na Tabela 2, é apresentada a estrutura de parentesco entre os animais analisados para as características GMD e D160. Verifica-se que, dos 507 touros, apenas 135 foram pais de vacas (avôs maternos) que desmamaram pelo menos um bezerro. Assim, aproximadamente $70 \%$ dos touros não tiveram o efeito materno expresso, por meio de suas filhas, neste conjunto de dados.

Tabela 1 - Estrutura dos dados no arquivo estudado para as características ganho médio diário do nascimento à desmama (GMD) e dias para ganhar $160 \mathrm{~kg}$ do nascimento à desmama (D160)

Table 1 - Characteristics of data structure for average daily gain from birth to weaning (ADG) and number of days to gain $160 \mathrm{~kg}$ from birth to weaning (D160)

\begin{tabular}{lc}
\hline Característica & GMD/D160 \\
Trait & ADG/D160 \\
\hline Número de registros & 16.592 \\
Number of records & \\
Número de animais com registro & 16.592 \\
Number of the animals with records & \\
Número de touros 1 & 507 \\
Number of sires ${ }^{1}$ & \\
Número de vacas & \\
Number of dams & \\
1 bezerro & 10.195 \\
calf & \\
2 bezerros & 6.240 \\
calves & \\
3 bezerros & 2.419 \\
4 bezerros & \\
5 bezerros & 960 \\
6 bezerros & 352 \\
7 bezerros & 151 \\
8 bezerros & 49 \\
9 bezerros & 18 \\
Número de grupos de contemporâneos & 3 \\
Number of contemporary group & 3 \\
Número de animais em A & 1.744 \\
Number of animals in A & \\
\hline
\end{tabular}

\footnotetext{
${ }_{1}^{1}$ Número de touros que possuem progênies com dados.

${ }^{1}$ Number of sires that had progeny with records.

${ }^{2}$ Número de vacas que possuem progênies com dados na primeira linha, nas linhas subseqüentes o número de grupos de irmãos maternos.

2 Number of dams wiht progeny in the data set. The following numbers are maternal sib group size.

A: matriz de parentesco.

A: relationship matrix.
}

A distribuição do GMD, verificada graficamente na Figura 1, aproxima-se de uma distribuição normal, ao passo que a característica D160 não apresenta esse tipo de distribuição, em virtude, principalmente, dos valores superiores e bastante extremos de D160, produzidos por GMDs pequenos, fazendo com que D160 apresente distribuição assimétrica (Figura 1). Métodos para a estimação de componentes de variância baseados na média aritmética das distribuições marginais, ao invés do vetor de modas da distribuição conjunta (como no caso do REML), são sugeridos por
Gianola \& Foulley (1990) e, possivelmente, seriam mais apropriados para características com distribuição assimétrica, como D160.

\section{Resultados e Discussão}

Na Tabela 3, são apresentados as médias, os desvios-padrão, o coeficiente de variação e os valores máximos e mínimos para GMD e D160.

A média observada para GMD neste estudo $(0,495 \mathrm{~kg} / \mathrm{dia})$ foi próxima àquela verificada por 
Tabela 2 - Estrutura de parentesco entre os animais no conjunto de dados analisado

Table 2 - Relationship structure on analysed data set

\section{Parentesco}

Relationship

Avôs paternos (pais de touros)

Total

22

Sire-paternal grandsires

Com desempenho próprio

With own performance record

Número médio de progênie

Average number of progeny

\begin{tabular}{|c|c|c|}
\hline \multirow{2}{*}{$\begin{array}{l}\text { Avôs maternos (pais de vacas) } \\
\text { Sire-maternal grandsires }\end{array}$} & Total & 135 \\
\hline & $\begin{array}{l}\text { Com desempenho próprio } \\
\text { With own performance record } \\
\text { Número médio de progênie } \\
\text { Average number of progeny }\end{array}$ & $\begin{array}{c}9 \\
6,81\end{array}$ \\
\hline \multirow{2}{*}{$\begin{array}{l}\text { Pais (touros) } \\
\text { Sires }\end{array}$} & Total & 507 \\
\hline & $\begin{array}{l}\text { Com desempenho próprio } \\
\text { With own performance record } \\
\text { Número médio de progênie } \\
\text { Average number of progeny }\end{array}$ & $\begin{array}{c}22 \\
32,73\end{array}$ \\
\hline \multirow{2}{*}{$\begin{array}{l}\text { Mães (vacas) } \\
\text { Dams }\end{array}$} & Total & 10.195 \\
\hline & $\begin{array}{l}\text { Com desempenho próprio } \\
\text { With own performance record } \\
\text { Número médio de progênie } \\
\text { Average number of progeny }\end{array}$ & 1,63 \\
\hline
\end{tabular}
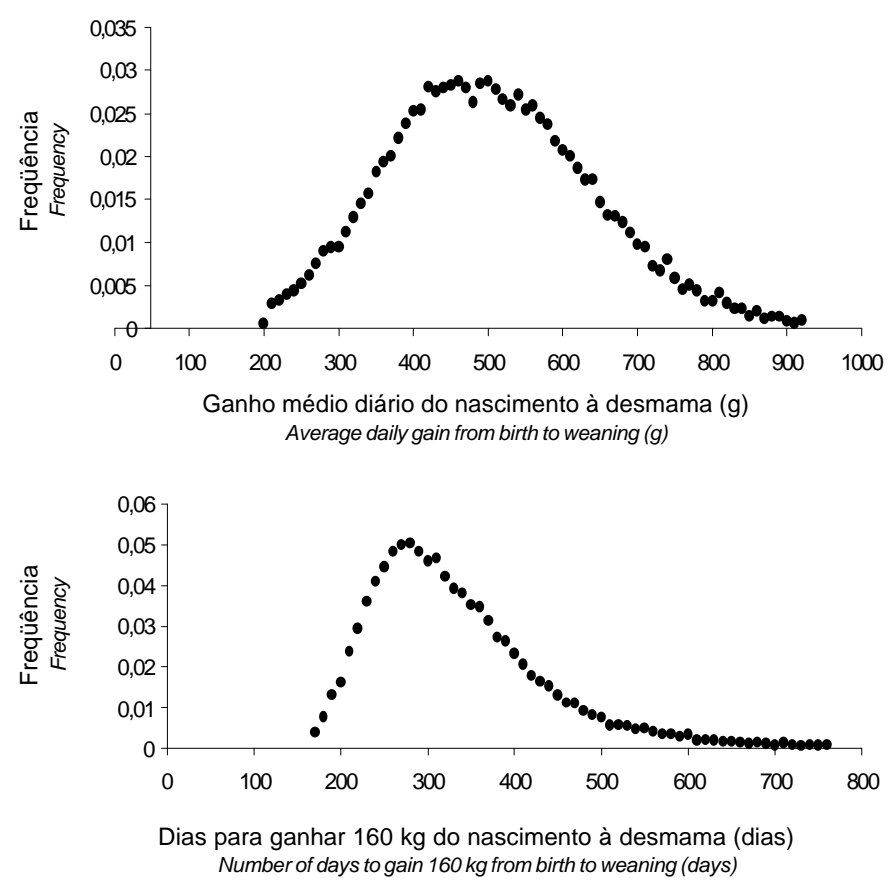

Figura 1 - Distribuição das freqüências dos dados para ganho médio diário do nascimento à desmama e dias para ganhar $160 \mathrm{~kg}$ do nascimento à desmama.

Figure 1 - Frequencies of data distribution for average daily gain from birth to weaning and number of days to gain $160 \mathrm{~kg}$ from birth to weaning.
Bacalhau et al. (1998), de 0,437 kg/dia, e por Silva et al. (1998), de 0,504 kg/dia, ambos em bovinos da raça Gir. $\mathrm{O}$ ganho médio diário do nascimento à desmama nesta raça, é menor que na raça Nelore. Silva et al. (1998) verificaram $0,630 \mathrm{~kg} /$ dia para a raça Nelore, utilizando dados de 423.174 animais. Valores próximos $(0,630 \mathrm{~kg} / \mathrm{dia}$ e $0,680 \mathrm{~kg} / \mathrm{dia})$ foram observados também, para a raça Nelore, por Muniz \& Queiroz (1998) e Paz et al. (1999), respectivamente. Deve-se considerar, entretanto, que parte das vacas Gir foi ou pode ter sido explorada para produção de leite, o que pode refletir no menor GMD.

Os bezerros Gir necessitariam, em média, de 353 dias para alcançar $160 \mathrm{~kg}$ do nascimento à desmama. Com base na média aritmética do GMD, um bezerro Gir com peso padronizado para 205 dias seria desmamado com $129,48 \mathrm{~kg}, 61 \mathrm{~kg}$ de diferença para $190 \mathrm{~kg}$, considerando o peso ao nascimento de $30 \mathrm{~kg}$ (Tabela 3).

Ortiz Peña et al. (2004) obtiveram 250 dias, em média, para a raça Nelore ganhar $160 \mathrm{~kg}$ do nascimento à desmama. Valores semelhantes (245 dias, em média) foram obtidos por Del Valle Garnero et al. (2001). 
Tabela 3 - Média, desvio-padrão (DP), coeficiente de variação (CV) e valores máximo e mínimo das características ganho médio diário do nascimento à desmama (GMD) e dias para ganhar $160 \mathrm{~kg}$ do nascimento à desmama (D160)

Table 3 - Mean, standard deviation (SD), coefficient of variation (CV), minimum and maximum values for average daily gain from birth to weaning (ADG) and number of days to gain $160 \mathrm{~kg}$ from birth to weaning (D160)

\begin{tabular}{lccccc}
\hline $\begin{array}{l}\text { Característica } \\
\text { Trait }\end{array}$ & $\begin{array}{c}\text { Média } \\
\text { Mean }\end{array}$ & $\begin{array}{c}\text { DP } \\
\text { SD }\end{array}$ & CV $(\%)$ & $\begin{array}{c}\text { Valor mínimo } \\
\text { Minimum value }\end{array}$ & $\begin{array}{c}\text { Valor máximo } \\
\text { Maximum value }\end{array}$ \\
\hline GMD (kg/dia) & 0,495 & 0,14 & 28,1 & 0,11 & 1,3 \\
$\begin{array}{l}\text { ADG (kg/day) } \\
\text { D160 (dias) }\end{array}$ & 353 & 119 & 33,7 & 123 & 1.521 \\
$D 160$ (days) & & & & & \\
\hline
\end{tabular}

Tabela 4 - Estimativas de componentes de variância e parâmetros genéticos e ambientais para ganho médio diário do nascimento à desmama (GMD) e dias para ganhar $160 \mathrm{~kg}$ do nascimento à desmama (D160), utilizando-se os dois modelos

Table 4 - Estimates of variance components and genetic and environmental parameter for average daily gain from birth to weaning (ADG) and number of days to gain $160 \mathrm{~kg}$ from birth to weaning (D160), using two models proposed

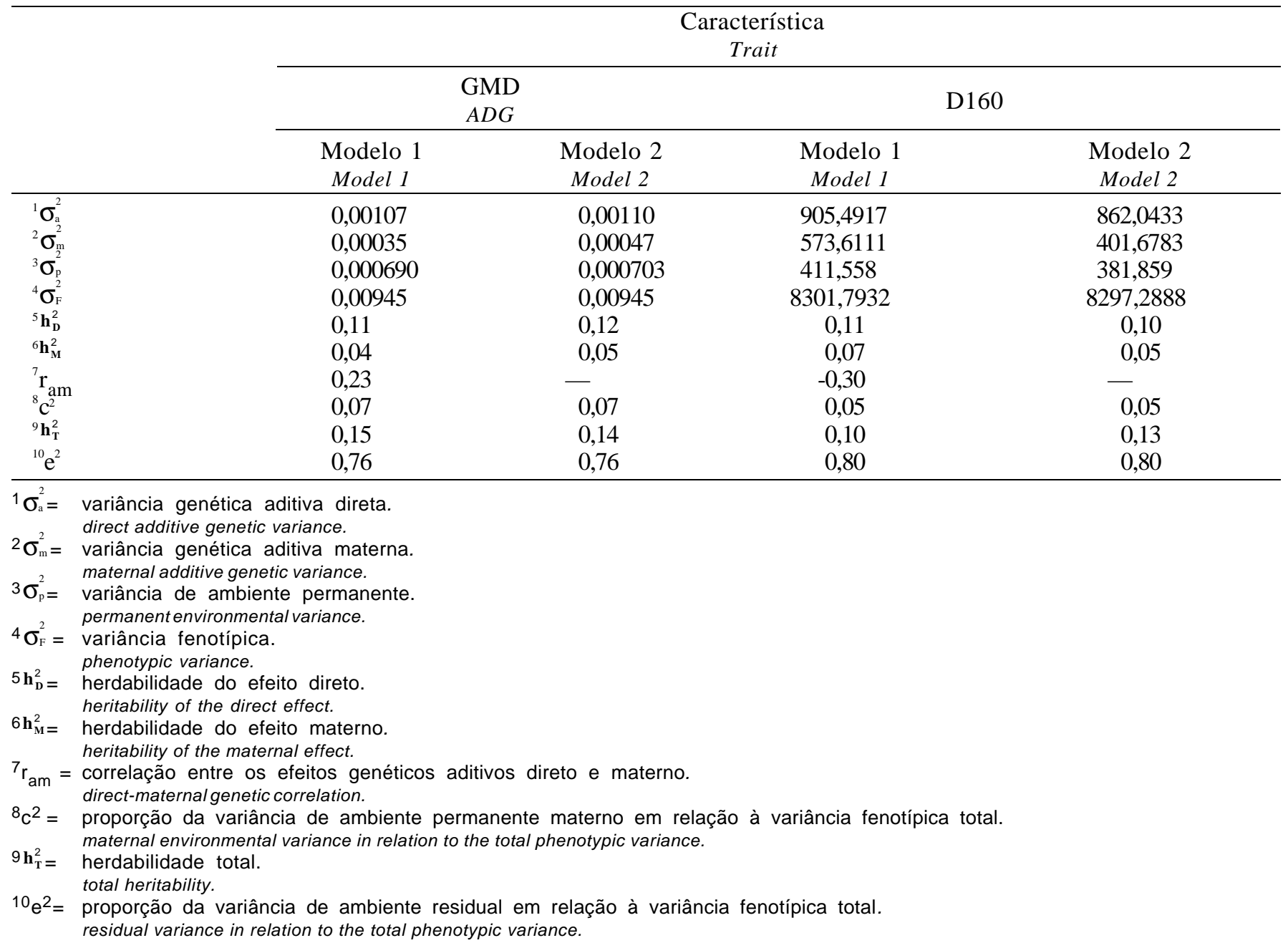

Ao calcular-se D160, utilizando-se a média aritmética do ganho médio diário no período prédesmama, relatada por Bacalhau et al. (1998), obtémse uma aproximação de 366 dias, em média, para D160. Da mesma forma, são obtidos 317 dias para os dados de ganho médio diário na pré-desmama apresentados em Silva et al. (1998), para bezerros Gir.
Assim, os resultados apresentados na literatura para D160 foram próximos aos encontrados neste estudo para a raça Gir. Verifica-se que a raça está um pouco distante do ideal: produzir um bezerro de 205 dias com $190 \mathrm{~kg}$, ao considerar-se D160 como critério de seleção, ou seja, seria necessário GMD de $0,7850 \mathrm{~kg} /$ dia. Neste conjunto de dados, somente cerca de, $2,3 \%$ 
dos animais avaliados atingiram $160 \mathrm{~kg}$ do nascimento à desmama, com 205 dias de idade. Entretanto, devese considerar que a raça Gir é menor que a Nelore e D160, com certeza, não é o critério mais adequado, sendo indicado definir a unidade de comercialização, isto é, peso e número de dias para atingir esse peso no período da pré-desmama, específica para a raça. $\mathrm{Na}$ Tabela 4, são apresentadas as estimativas dos parâmetros genéticos e de ambiente obtidos para as características de crescimento na pré-desmama estudadas, conforme os dois modelos propostos.

Os coeficientes de herdabilidade do efeito direto estimados para GMD e D160 foram iguais para o modelo 1. Quando foi considerada a covariância entre efeitos genéticos materno e direto como zero $\left(\sigma_{\mathrm{am}}\right)$, modelo 2 , as estimativas dos parâmetros genéticos foram similares e as estimativas de herdabilidade do efeito direto para GMD e D160, iguais a 0,12 e 0,10 , respectivamente. Estes resultados sugerem que a seleção para GMD e D160 proporcionaria ganhos genéticos equivalentes.

A contribuição do efeito genético materno para a variação total das características analisadas foi de menor magnitude, indicando que a habilidade materna teve menor importância que o efeito direto para as características da pré-desmama neste conjunto de dados. As estimativas das variâncias de ambiente permanente das características estudadas, GMD e D160, não foram alteradas quando utilizados os diferentes modelos. A contribuição deste componente foi pouco mais expressiva para GMD (7\%) (Tabela 4).

Segundo Eler et al. (1995), os efeitos de ambiente permanente pós-natal, em gado de corte, decorrem de causas que afetam todos os registros da progênie para uma mesma vaca, a exemplo de acidentes no úbere que poderiam criar diferenças permanentes entre vacas. Nesses casos, a expressão das diferenças entre vacas, possivelmente, deriva de diferenças em produção de leite, além do efeito da própria vaca.

Embora de pequena magnitude, a inclusão dos efeitos maternos no modelo é necessária, pois, de acordo com Meyer (1992), a não inclusão destes na análise levaria ao aumento da variância genética aditiva e, conseqüentemente, estimativas mais altas de herdabilidade direta seriam obtidas.

Os valores das estimativas de herdabilidade dos efeitos diretos e maternos foram menores que aqueles obtidos por Fries et al. (1996), para a raça Hereford, e Ortiz Peña et al. (2004) e Marcondes et al. (2000), para a raça Nelore, estudando as mesmas características. Entretanto, deve-se considerar que as estimativas dos parâmetros genéticos podem apresentar grande variabilidade, por serem obtidas mediante diferentes métodos estatísticos e para diferentes populações.

Mesmo com estimativas de herdabilidade de pequena magnitude, existiu variabilidade de origem genética nestas características, o que possibilitaria melhorar a velocidade de crescimento na pré-desmama, utilizando-se tanto GMD como D160.

A correlação genética entre os efeitos aditivos direto e materno $\left(\mathrm{r}_{\mathrm{am}}\right)$ foi um pouco maior e negativa para D160, indicando que as vacas com maior habilidade materna desmamariam bezerros com menor velocidade de crescimento e necessitariam de maior número de dias para atingir os requeridos $160 \mathrm{~kg}$. Para GMD, $\mathrm{r}_{\mathrm{am}}$ foi de menor magnitude e positiva, ou seja, vacas de melhores habilidades maternas estariam desmamando bezerros com maiores taxas de crescimento. Entretanto, os valores estimados $(0,23 \mathrm{e}-0,30)$ indicam associação fraca entre as características, sugerindo pequeno impacto no progresso genético, caso a seleção seja aplicada em qualquer uma delas.

$\mathrm{Na}$ Tabela 5, podem ser verificados, para as características GMD e D160, aumento no valor de -2 $\log \mathrm{L}$ e a significância desse aumento, com a retirada de um parâmetro do modelo $\left(\sigma_{\mathrm{am}}=0\right)$. A diferença entre os modelos não foi significativa, ou seja, modelos com $\sigma_{\mathrm{am}}=0$ e $\sigma_{\mathrm{am}} \neq 0$ foram equivalentes, e considerar $\sigma_{\mathrm{am}}=0$ é uma boa alternativa no caso deste conjunto de dados.

A existência da covariância negativa entre os efeitos aditivos direto e materno, poderia ser atribuída à "fase de desequilíbrio gamético" ou "Efeito Bulmer" (Falconer \& Mackay, 1996). Considerandose dois genes diferentes, afetando determinada característica, e a seleção com base na soma dos efeitos genéticos em ambos os locos, um animal com valor alto para o loco um teria oportunidade de ser selecionado, independentemente de seu valor para o loco dois. Da mesma forma, um animal com alto valor para o loco dois poderia ser selecionado, independentemente de seu valor para o loco um. Animais com valor moderadamente alto para o loco 1 seriam selecionados se o valor para o loco 2 fosse também moderadamente alto. Assim, de acordo com Gibson \& Van Arendonk (1998), como consequiência, o efeito dos genes nos dois locos é negativamente correlacionado nos indivíduos selecionados. 
Tabela 5 - Comparação dos modelos $1\left(\sigma_{a m} \neq 0\right)$ e $2\left(\sigma_{a m}=0\right)$ pelo teste de razão de verossimilhança Table 5 - Comparison of models $1\left(\sigma_{a m^{\neq}} 0\right)$ and $2\left(\sigma_{a m}=0\right)$, using likelihood ratio test $(-2 \log L)$

\begin{tabular}{cc} 
Característica & Aumento do -2 log L \\
Trait & $\begin{array}{c}\text { Modelo 2 - Modelo 1 } \\
\text { Model 2 - Model 1 }\end{array}$ \\
GMD & $0,24 \mathrm{~ns}$ \\
ADG & $0,70 \mathrm{~ns}$ \\
\hline
\end{tabular}

\footnotetext{
- $2 \log L$ do modelo 2, expresso como desvio do $-2 \log L$ do modelo 1 , sendo $L$ o valor da função de verossimilhança. ns: não-significativo, com probabilidade de erro de $5 \%$.

(-2 $\log L$ of model 2) $-(-2 \log L$ of model 1$), L$ is the likehood function.

$n s$ : Not significant at $5 \%$ probability.
}

As estimativas de pequena magnitude para $\mathrm{r}_{\mathrm{am}}$, negativa para D160 e positiva para GMD obtidas pelo Modelo 1, poderiam estar, também, relacionadas ao tamanho do conjunto de dados e sua estrutura. Meyer (1998) salientou que este resultado poderia ser conseqüência de problemas associados à estrutura dos dados, heterogeneidade de variâncias e, em certos casos, má formação de grupos de contemporâneos, pois $r_{a m}$ negativas não são verificadas e dados experimentais, além da pressuposição de ausência de covariância entre efeitos genéticos e ambientais permanentes.

No presente trabalho, deve-se considerar a estrutura fraca de família, uma vez que 70\% dos touros não foram pais de vacas que desmamaram pelo menos um bezerro. Para estes touros, a correlação entre efeito direto e materno é exatamente -1 . Como consequiência, o melhor touro para o efeito direto é o pior para o efeito materno, enquanto o pior para o efeito direto é o melhor para o efeito materno (Schaeffer, 1993).

Uma estimativa positiva para $\mathrm{r}_{\mathrm{am}}$ seria mais aceitável que justificar a existência de antagonismo genético entre efeitos. As vacas com maiores produções de leite seriam mães dos melhores touros, o que seria, provavelmente, mais verdadeiro que concluir que as melhores vacas seriam mães dos piores touros.

Assim, é melhor seguir a recomendação de Schaeffer (1996), citado por Fries \& Albuquerque (1998), de se usar covariância igual a zero entre os efeitos genéticos direto e materno, ou seja, os resultados suportam o uso do modelo com efeitos direto, materno e de ambiente permanente (Modelo 2), em vez de todos os efeitos do anterior, e ainda estrutura de covariâncias entre os efeitos direto e materno (Modelo1), quando (ou enquanto) estimativas duvidosas da $r_{\text {am }}$ são obtidas.

A contribuição da variância residual, como proporção da variância fenotípica total $\left(\mathrm{e}^{2}\right)$, foi de $76 \%$ para GMD e de $80 \%$ para D160. O conjunto de dados utilizado neste estudo continha informações de bovinos criados sob diversas condições, em várias regiões do Brasil. Os altos valores verificados para a variância residual indicaram a necessidade da melhoria do ambiente de criação, assim como a padronização e formação de lotes de manejo, o que refletiria na possibilidade de melhor definição de grupo de contemporâneos. Os resultados indicaram que a velocidade de crescimento em bovinos da raça Gir pode ser modificada por meio da seleção, considerando qualquer uma das características, GMD ou D160.

\section{Conclusões}

Modelos de análise considerando $\left(\sigma_{\mathrm{am}} \neq 0\right)$ ou não $\left(\sigma_{\mathrm{am}}=0\right)$ a estrutura de covariância genética entre efeitos direto e materno foram equivalentes nas análises do ganho médio diário do nascimento à desmama e de dias para atingir $160 \mathrm{~kg}$ do nascimento à desmama.

Apesar das estimativas de herdabilidade de pequena magnitude, houve variabilidade de origem genética em ambas as características, o que possibilitaria melhorar a velocidade de crescimento pré-desmama, utilizando-se GMD ou D160 como critério de seleção.

Os altos valores verificados para a variância residual indicam a necessidade da melhoria do ambiente de criação, assim como a padronização e formação de lotes de manejo, o que traria reflexos na melhor definição de grupos de contemporâneos ou exigiria maiores cuidados na coleta dos dados, principalmente em relação à correta identificação do manejo dos bezerros e vacas, tendo em vista que parte dessas vacas explorada também para produção de leite.

Ao se estudar o número de dias necessários para o animal atingir $160 \mathrm{~kg}$ do nascimento à desmama, verifica-se a necessidade da definição de uma meta, em termos de $\mathrm{kg}$ a atingir do nascimento à desmama, mais factível para a raça Gir. 


\section{Agradecimento}

À Associação Brasileira de Criadores de Zebu (ABCZ), pela cessão dos dados do arquivo da raça Gir

\section{Literatura Citada}

BACALHAU, A.; GUEDES, P.L; NOVAIS, L.P. et al. Fontes de variação de pesos e ganhos de pesos até o desmame de bezerro Gir. In: REUNIÃO ANUAL DA SOCIEDADE BRASILEIRA DE ZOOTECNIA., 35., 1998, Botucatu. Anais...Viçosa, MG: Sociedade Brasileira de Zootecnia, 1998. v3, p.389-391.

BOLDMAN, K.G.; KRIESE, L.A.; Van VLECK, L.D. A manual for use MTDFREML. Nebrasca: Clay Center; USDA-ARS, 1993. $120 \mathrm{p}$.

CARDOSO, F.F.; CARDELINO, R.A.; CAMPOS, L.T. Componentes de (Co) variância e parâmetros genéticos para caracteres produtivos à desmama de bezerros Angus criados no estado do Rio Grande do Sul. Revista Brasileira de Zootecnia, v.30, n.1, p.41-48, 2001.

DEL VALLE GARNEIRO, A.; LÔBO, R.B.; BEZERRA, L.A.F. et al. Comparação de alguns critérios de seleção para crescimento na raça Nelore. Revista Brasileira de Zootecnia, v.30, n.3, p.714-718, 2001.

ELER, J.P.; Van VLECK, L.D.; FERRAZ, J.B. et al. Estimation of variances due to direct and maternal effects for growth traits of Nellore catlle. Journal of Animal Science, v.73, p.3253-3258, 1995.

FALCONER, D.S.; MACKAY, T.F.C. Introduction to qantitative genetics. 4.ed. Longman: 1996. 464p.

FRIES, L.A. Cruzamentos em gado de corte. In: SIMPÓSIO SOBRE PECUÁRIA DE CORTE, 4., 1996, Piracicaba. Anais... Piracicaba: Fundação de Estudos Agrários, 1996.

FRIES, L.A.; BRITO, F.V.; ALBUQUERQUE, L.G. Possíveis conseqüências na seleção para incrementar pesos às idadespadrão vs. reduzir idades para produzir unidades de mercado. In: REUNIÃO DA SOCIEDADE BRASILEIRA DE ZOOTECNIA, 30., 1996, Fortaleza. Anais... Fortaleza: Sociedade Brasileira de Zootecnia, 1996. p.310-312.

FRIES, L.A.; ROSO, V.M. Conectabilidade em avaliações genéticas de gado de corte: uma proposta heurística. In: REUNIÃO ANUAL DA SOCIEDADE BRASILEIRA DE ZOOTECNIA, 34., 1997, Juiz de Fora. Anais... Juiz de Fora: Sociedade Brasileira de Zootecnia, 1997. p.159-161.

FRIES, L. A. Connectability in beef cattle evaluation: the heuristic approach used in MILC. FOR. In: WORLD CONGRESS ON GENETICS APPLIED TO LIVESTOCK PRODUCTION, 6 , 1998, Armidale. Proceedings... Australia: 6WCGALP, 1998. v.27, p.449-450.

FRIES, L.A.; ALBUQUERQUE, L. G. Pressuposições e restrições dos modelos animais com efeitos maternos em gado de corte. In: COSTA, M.J.; CROMBERG, V.U. (Eds.) Comportamento materno em mamíferos: bases teóricas e aplicações aos ruminantes domésticos. Jaboticabal: SBEtETCO, 1998. p.179-214.

GIANOLA, D.; FOULLEY, J.L. Variance estimation from integrated likelihood (VEIL). Genetic, Selection, Evolution, v.22, p.403-417, 1990.

GIANNONI, M.A.; GIANNONI, M.L. Genética e melhoramento de rebanhos nos trópicos. 2.ed. São Paulo: Nobel, 1989. 463p.
GIBSON, J.P.; Van ARENDONK, J. Introduction to the design of animal breeding strategies. Poland: Warsaw Agricultural University, 1998. 259p. (Lectures notes)

HOOG, R.V.; CRAIG, A.T. Introduction to mathematical statistics. 5.ed. New Jersey: Prentice Hall, 1995. 564p.

MARCONDES, C.R.; BERGMANN; J.A.G.; ELER, J.P. et al. Análise de alguns critérios de seleção para características de crescimento na raça Nelore. Arquivo Brasileiro de Medicina Veterinária e Zootecnia, v.52, n.1, p.83-89, 2000.

MEYER, K. Uso do DFREML. Notas do WorkShop, Departamento de Melhoramento Animal, Jaboticabal: FCAV/ UNESP, 1998.

MEYER, K. Variance components due to direct and maternal effects for growth traits of Australian beef cattle. Livestock Production Science, v.31, p.179-204, 1992.

MUNIZ, C.A.S.D.; QUEIROZ, S.A. Avaliação do peso à desmama e do ganho médio de bezerros cruzados, no estado do Mato Grosso do Sul. Revista Brasileira de Zootecnia, v. 27, n.3, p.504-512, 1998.

MUNIZ, C.A.S.D.; CARVALHEIRO, R.; FRIES, L.A. et al. Critérios de seleção baseados em médias aritméticas e em médias harmônicas. In: REUNIÃO ANUAL DA SOCIEDADE BRASILEIRA DE ZOOTECNIA, 38., 2001, Piracicaba. Anais... Piracicaba: Sociedade Brasileira de Zootecnia, 2001. p.629.

ORTIZ PEÑA, C.D.; CARVALHEIRO, R.; QUEIROZ, S.A. et al. Comparison of selection criteria for pre-weaning growth traits of Nelore cattle. Livestock Production Science, v.86, p.163-167, 2004.

PAZ, C.C.; ALBUQUERQUE, L.G.; FRIES, L.A. Fatores de correção para ganho de peso médio diário no período do nascimento ao desmame em bovinos raça Nelore. Revista Brasileira de Zootecnia, v.28, n.1, p.65-73. 1999.

PELICIONI, L.C.S; PASCOA, L.; MUNIZ, C.A.S.D. et al. Efeito da idade da vaca ao parto e da data juliana de nascimento sobre características pré-desmama de bezerros da raça Gir. Revista Brasileira de Zootecnia, v.31, n.1, p.61-70, 2002.

PIMENTA FILHO, E.C.; MARTINS, G.A.; SARMENTO, J.L.R. et al. Estimativa de herdabilidade para efeitos direto e materno de características de crescimento de bovinos Guzerá no estado da Paraíba. Revista Brasileira de Zootecnia, v.30, n.4, p.1220-1223, 2001.

RIBEIRO, M.N.; PIMENTA FILHO, E.C.; MARTINS, G.A. et al. Herdabilidade para efeitos direto e materno de características de crescimento de bovinos Nelore no estado da Paraíba. Revista Brasileira de Zootecnia, v.30, n.4, p.1224-1227, 2001.

ROBINSON, D. L. Estimation and interpretation of direct and maternal genetic parameters for weights of Australian Angus cattle. Livestock Production Science, v.45, p.1-11, 1996.

SCHAEFFER, L.R. Linear models and computing strategies in animal breeding [mimeo]. Ghelph: University of Guelph, 1993.

SILVA, L.O.; EUCLIDES FILHO, K.; FIGUEIREDO, G.R. et al. Genetic Trend in zebu (Bos Indicus) breeds in Brazil. In: WORLD CONGRESS ON GENETICS APPLIED TO LIVESTOCK PRODUCTION, 6., 1998, Armidale. Proceedings... Australia: 6WCGALP, 1998. v.23, p.137-140.

WILLHAM, R.L. The role of maternal effects in animal breeding: III - Biometrical aspects of maternal effects in animals. Journal of Animal Science, v.35, p.1288-1302, 1972.

Recebido em: 22/04/03 Aceito em: 28/01/05 